Décadrages Décadrages

cınéma, à travers champs Cinéma, à travers champs

11 | 2007

Terrence Malick

\title{
«Le Nouveau Monde » ou la vie dans les bois : la culture du transcendantalisme dans les films de Terrence Malick
}

François Bovier

\section{(2) OpenEdition \\ Journals}

Édition électronique

URL : http://journals.openedition.org/decadrages/401

DOI : $10.4000 /$ decadrages.401

ISSN : 2297-5977

Éditeur

Association Décadrages

Édition imprimée

Date de publication : 1 octobre 2007

Pagination : 65-73

ISBN : 978-2-9700582-6-7

ISSN : 2235-7823

Référence électronique

François Bovier, « «Le Nouveau Monde » ou la vie dans les bois : la culture du transcendantalisme dans les films de Terrence Malick », Décadrages [En ligne], 11 | 2007, mis en ligne le 01 octobre 2008, consulté le 19 avril 2019. URL : http://journals.openedition.org/decadrages/401 ; DOI : 10.4000/ decadrages.401 


\section{"Le Nouveau Monde" ou la vie dans les bois:}

\section{la culture du transcendantalisme dans les films}

\section{de Terrence Malick}

\section{par François Bovier}

Le rapport de Terrence Malick au transcendantalisme ou, plus précisément, aux écrits de Ralph Waldo Emerson, Henry David Thoreau et Walt Whitman sur la nature et la culture des pionniers américains constitue un cliché dans la littérature critique qui porte sur ses films ${ }^{\mathbf{1}}$. C'est ce stéréotype que nous prendrons en considération, en nous concentrant sur son dernier film, The New World (Le Nouveau Monde, 2005), qui thématise la relation heureuse de l'homme primitif à la terre nourricière et, inversement, l'inadéquation du comportement des pionniers qui «arraisonnent» la nature par la technique ${ }^{2}$ en exploitant une terra incognita. Cepandant, cette opposition entre nature et culture est déjà présente dans les films précédents de Malick, où la relation de l'homme à la nature constitue seulement l'arrière-plan de l'action, celle-ci réactivant des genres cinématographiques fortement codifiés.

\section{De la nature}

Dans Badlands (La Balade sauvage, 1973), la nature confine au chromo: les héros fuient en voiture le long de la ligne d'horizon au coucher du soleil, dans une parodie de Bonnie and Clyde désœuvrés; les gros plans égrenés sur la faune et la flore de la forêt, bientôt prise d'assaut par les forces de l'ordre, pastichent le style du National Geographic, dont un numéro est consulté par le héros, juché dans une cabane bâtie sur un arbre, dont l'accès est protégé par des pièges. Dans Days of Heaven (Les Moissons du ciel, 1978), le rapport à la nature est duel, puisqu'il est médiatisé par les différences de classes sociales: le vent qui agite les champs constitue une image bucolique pour le grand propriétaire terrien (qui scrute à la lorgnette l'une de ses employées, dont il s'énamoure); pour la classe laborieuse, le champ est associé à une automatisation des gestes, une précision comparable au travail à l'usine (les gestes de Richard Gere
1 "L'influence du transcendantalisme de Thoreau, Emerson et Whitman est omniprésente dans le cinéma de Terrence Malick, et nombre de commentateurs l'ont justement remarqué. [...] L'opposition entre la nature, imperturbable et éternelle (voir la contre-plongée finale du Nouveau Monde ou les perroquets multicolores pendant l'avancée des soldats dans La Ligne rouge), et la technique, si manifeste depuis $L a$ Balade sauvage, est au fondement de ce geste cinématographique: champs/fonderie dans Les Moissons du ciel, faune et flore/combats dans La Ligne rouge, simplicité adamique/civilisation raffinée dans Le Nouveau Monde. "(JeanMichel Durafour, "Le Nouveau Monde de Terrence Malick: quelques propositions pour un cinéma de l'anti-regard", Cadrage, mars 2006, www.cadrage.net/).

2 "Le dévoilement qui régit la technique moderne est une pro-vocation [Herausfordern] par laquelle la nature est mise en demeure de livrer une énergie qui puisse comme telle être extraite [herausgefordert] et accumulée. [...] L'écorce terrestre se dévoile aujourd'hui comme bassin houiller, le sol comme entrepôt de minerais. Tout autre apparaît le champ que le paysan cultivait autrefois, alors que cultiver [bestellen] signifiait encore: entourer de haies et entourer de soins. Le travail du paysan ne pro-voque pas la terre cultivable. Quand il sème le grain, il confie la semence aux forces de croissance et il veille à ce qu'elle prospère. Dans l'intervalle, la culture des champs, elle aussi, a été prise dans le mouvement aspirant d'un mode de culture [Bestellen] d'un autre genre, qui requiert [stellt] la nature. II la requiert au sens de la provocation. L'agriculture est aujourd'hui une industrie d'alimentation motorisée." (Martin Heidegger, "La question de la technique" [1953], Essais et Conférences, Gallimard, Paris, 1980, p. 11 [traduit par André Préau]). 
3 "Je suppose que quiconque s'est intéressé à ce film a envie de comprendre au service de quoi ses sommets de beauté se sont inscrits; [...]. De quelque manière que l'on comprenne son sujet, nul n'aurait pu entreprendre de l'explorer sans la confiance solide que sa capacité à extraire de la beauté de la nature, et de la projection ou du déplacement photographique de la nature, était inépuisable, ce qui est bien sûr avoir confiance en même temps dans la capacité de la nature et du cinéma à fournir de la beauté. [...] Tenterons-nous d'exprimer le sujet comme celui où les travaux, les émotions et les imbrications des êtres humains sont à tout moment réduits à l'insignifiance par les tours désinvoltes de la terre et du ciel?" (Stanley Cavell, La Projection du monde, Belin, Paris, 1999 [1979], pp. 12-13 [traduit par Christian Fournier].) Rappelons que Malick est un ancien étudiant de Cavell, et qu'il a enseigné la philosophie au MIT et traduit plusieurs textes de Heidegger.

4 Whitman lui-même oppose la raison du progrès et de l'industrie au désordre et à la furie de la guerre: "Loin de nous les thèmes de guerre! Loin de nous la guerre elle-même! / Hors de ma vue frémissante pour ne revenir jamais plus cet étalage de cadavres noircis, mutilés! / Cet enfer déchaîné, ce débordement de sang, conviennent à des tigres sauvages, à des loups à la langue pendante, non point à des hommes doués de raison [...]." (Walt Whitman, "Chant de l'exposition" [1871], Poèmes. Feuilles d'herbe [1891-1892], Gallimard, Paris, 1992 [1918], p. 131 [traduit par Louis Fabulet]).

5 "Quelle est cette guerre au cœur de la nature? Pourquoi la terre affronte-t-elle la mer? La nature renforce-t-elle une force vengeresse? Non pas une force, mais deux?".

The Thin Red Line, 1998 dans la forge à l'ouverture du film sont rejoués lors de la moisson). Si la nature peut furtivement apparaître comme un lieu d'évasion et un espace du désir, elle est le plus souvent associée à une menace, à une puissance de mort: la vie dans les bois est réduite à un terrain de traque pour les amants en fuite, tandis que les champs sont dévastés par un nuage de sauterelles, avant de s'embraser. Comme le note Stanley Cavell, l'éloge de la beauté de la nature dans Days of Heaven comporte un revers, à savoir l'insignifiance de l'homme 3 .

The Thin Red Line (La Ligne rouge, 1998), en se concentrant sur les mécanismes de la violence guerrière ${ }^{4}$, appréhende la nature comme un obstacle à surmonter, comme un camouflage où se dissimule l'ennemi. Ce remake d'un film d'Andrew Marton (The Thin Red Line, 1964) comporte un prologue qui acquiert une position de relative autonomie par rapport aux événements relatés: le film s'ouvre sur un prélude heureux et innocent qui entretient d'étroites relations avec la première partie de The New World. Les îles du Pacifique Sud, avant de devenir le lieu d'affrontements entre les forces américaines et japonaises pendant la Seconde Guerre, apparaissent comme un cadre idyllique pour deux soldats qui se sont harmonieusement intégrés à la communauté indigène. The Thin Red Line, adoptant un rythme fluide et systématisant les fondus enchaînés, débute par des plans de nature sauvage: un alligator se glisse dans l'eau; la caméra parcourt des troncs d'arbres, baignés par les rayons du soleil et cadrés en abruptes contre-plongées, puis elle se fixe sur l'extrémité de branches qui se détachent sur le ciel. La voix-over d'un protagoniste du film résonne à travers les plans, interrogeant les forces obscures et duelles de la nature, leur duplicité5. Le film se poursuit en présentant les activités des indigènes, en particulier des enfants, et les rapports apaisés que l'un des soldats entretient avec la population. La

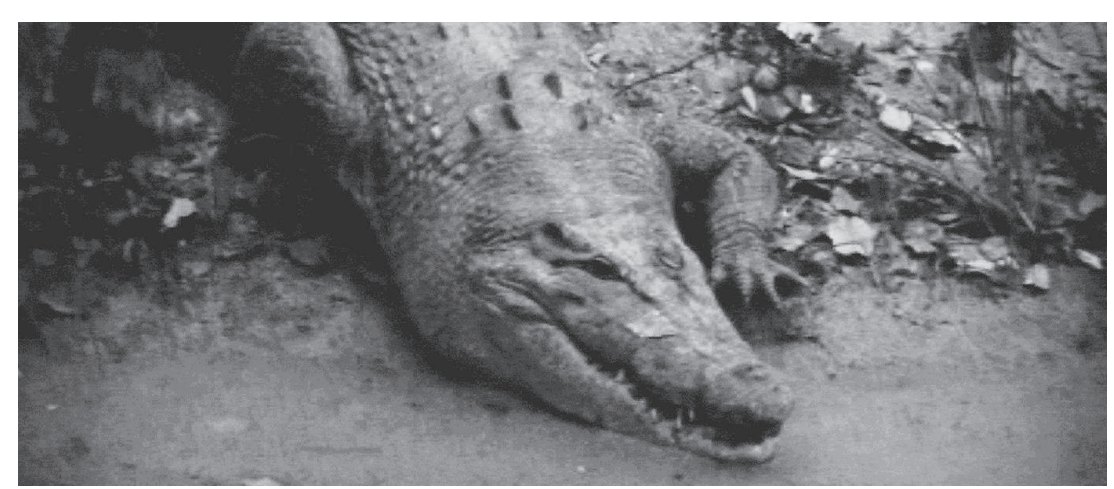



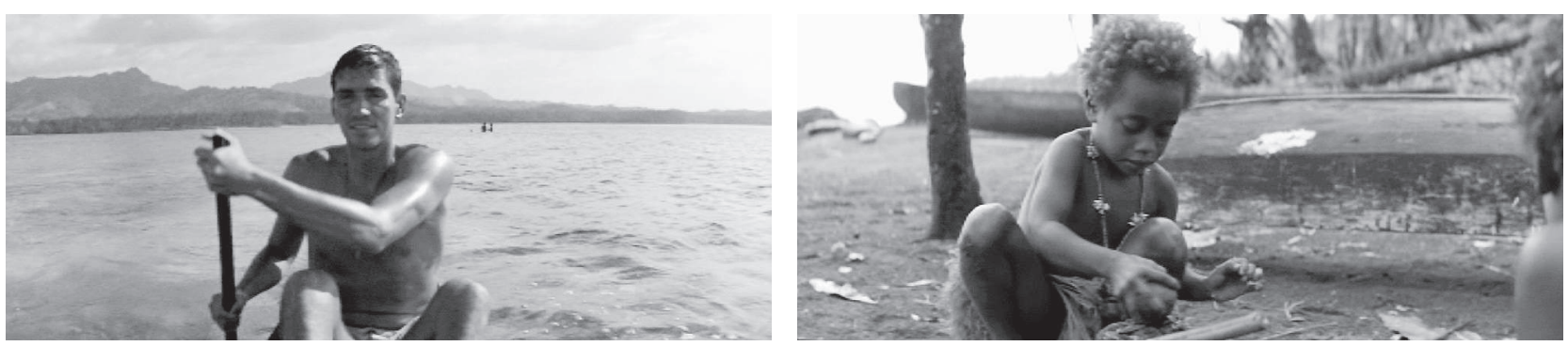

The Thin Red Line, 1998

mer et la rivière font ici figure de liens, images du liquide amniotique et fantasme d'une régression utérine. La caméra suit deux enfants qui nagent sous l'eau et qui traversent une barrière de corail; suite à un fondu enchaîné, celle-ci cadre la surface miroitante de l'eau, découvrant le soldat qui rame; un peu plus tard, la population se baigne dans une rivière, les femmes font la toilette de leurs enfants. En champs/contrechamps s'effectue une alternance entre les scènes indigènes et les plans rapprochés sur le protagoniste blanc. La générosité de cette terre hospitalière est soulignée par un plan à valeur allégorique : le soldat tient dans sa main une série de bigorneaux et de coquillages, fruits de la mer et de la nature. Mais ces scènes de communion entre le soldat et les indigènes sont interrompues par l'irruption d'un patrouilleur: visualisé du point de vue des indigènes, celui-ci provoque la fuite des habitants. La séquence suivante se centre sur le dialogue entre un sergent américain et le soldat déserteur enfermé dans les cales du bateau. Les propos tenus par le déserteur ("J'ai vu un autre monde. Parfois, je pense que c'est juste... mon imagination") anticipent la situation de retrait que le protagoniste affranchi de The New World occupe vis-à-vis de la société occidentale et de ses règles: l'homme libre est menacé par la civilisation, qui réduit au statut de chimère l'amour et les échanges heureux entre individus.

La morale de la fable, chez Malick, rejoint le propos et la thématique des westerns: qu'il s'agisse d'un film policier, d'un mélodrame ou d'un film de guerre, le territoire représente inévitablement un espace à conquérir (par les armes) ou à conserver (par le maintien de l'ordre). En un sens, le "tournant» que Jacques Rancière identifie dans les westerns d'Anthony Mann pourrait être appliqué à l'ensemble de la filmographie de Malick:

«Et c'est cela d'abord que pourrait signifier le 〈tournant indien> du western: non pas tant la découverte que les Indiens aussi sont des hommes qui pensent, aiment et souffrent, mais plutôt le sentiment que leur expropriation fait destin commun et interdit la romance qui ferait naître avec 〈sa〉 terre l'homme américain et ses vertus. [...] 
6 Jacques Rancière, La Fable cinématographique, Seuil, Paris, 2001, pp. 106-107, p. 113.

7 Nous citons les sous-titres de l'édition DVD du film.

8 Voir, par exemple, l'éloge suivant de la démocratie américaine et de son esprit communautariste: "Allons, je ferai que le continent devienne indissoluble, / Je ferai la plus splendide race sur laquelle jamais le soleil ait brillé, / Je ferai de divins pays magnétiques, / Avec l'amour des camarades, / Avec l'amour pour toute la vie des camarades. / Je planterai la camaraderie aussi serrée que des arbres le long de tous les fleuves d'Amérique, et le long des rives, des grands lacs et par toutes les prairies, / Je ferai inséparables les cités grâce à leurs bras passés autour du cou l'un de l'autre, / Grâce à l'amour des camarades, / Grâce à l'amour viril des camarades." (Walt Whitman, "Pour toi, ô démocratie ", Poèmes. Feuilles d'herbe, op. cit., p. 29).
La communauté manienne n'est pas de lieu, de famille ou d'institution. Elle est essentiellement de rencontre.» 6

Aussi le rapport de Malick au transcendantalisme se négocie-t-il toujours sur le mode de la nostalgie et de l'expropriation, la terre promise constituant un horizon hors d'atteinte, un paradis perdu. Ultime fantasme écologiste et dernière variation sur le western, The New World réactive l'économie du don, en l'opposant à la dynamique réifiante de l'usure, à l'usage de l'or et de la monnaie. Le film propose ainsi l'utopie d'une mise en commun des biens, une image romantisée d'une idylle qui ignore les avilissements de la civilisation. Ce point de vue, reconduisant la logique de l'atavisme, mine de l'intérieur la critique de la colonisation que Malick semble vouloir articuler.

\section{Natura naturans}

Dans The New World, Malick, pour reprendre la terminologie de Spinoza, oppose une "nature objet», construite et instrumentalisée (natura naturata), à une "nature sujet", productive et archaïque (natura naturans). Cette dualité est traversée par le clivage entre la civilisation anglaise et la société primitive du Nouveau Monde. Comme le constate l'équipage blanc qui débarque sur ces territoires, la nature est luxuriante, les eaux recelant des huîtres de la taille d'une main. Mais le fort qu'ils érigent, afin de se protéger des intempéries et des assauts de l'extérieur, deviendra leur tombeau: les Anglais connaissent la misère et la maladie, en cédant à la quête de l'or ("pro-voquant la terre») plutôt qu'en s'adonnant à l'agriculture ("confiant la semence aux forces de la croissance» - cf. note 2). Seul le renégat John Smith, envoyé à la recherche du roi de ce territoire sauvage, parviendra à s'intégrer. Lors de son expédition, dérivant au fil de l'eau, il investit sa mission comme la fondation d'une nouvelle culture, d'une société égalitaire et d'une justice fraternelle. Il égrène, en voix-over, un credo qui reconduit les valeurs du transcendantalisme:

«Nous prendrons un nouveau départ. Tout recommencera. Tous les bienfaits de la nature sont là. Personne ne sera pauvre. Il y a de la bonne terre pour tous. Il suffit de la travailler. Nous bâtirons une communauté. Dur labeur et autonomie la mèneront. Sans propriétaires pour extorquer le fruit de notre labeur.» $\mathbf{7}$

Nous reconnaissons là l'ethos de l'aventure solitaire de Thoreau dans les bois auprès de l'étang de Walden, ainsi que le communautarisme de Whitman chantant les bienfaits de l'Amérique ${ }^{8}$. Le parcours de Smith au cœur de la nature sauvage, marqué par la réduction des membres de l'expédition au fur et à mesure de son avancée, emprunte la structure $\mathrm{du}$ rite initiatique, le capitaine se dépouillant de tous ses biens pour 
renouer avec son être authentique. Mais ce volontarisme n'est pas suffisant: Smith est fait prisonnier. S'il échappe à la mort, c'est grâce à l'intervention d'une jeune indigène, Pocahontas; en fin de compte, c'est l'amour qui l'éveillera à la vie naturelle.

Malick mime, à travers une forme de cinéma lyrique, le rythme ample et heurté des vers libres de Whitman, leur syntaxe brisée et sans cesse relancée, ce flux d'incantations qui constitue une authentique tradition dans la poésie épique américaine. Mais contrairement au processus d'objectification d'un William Carlos Williams qui prône un retour aux choses ("no ideas but in things»9), ou d'un Charles Olson qui réinscrit le passé de la ville de Gloucester dans le récit de la fondation des Etats-Unis (John Smith est un personnage qui apparaît à plusieurs reprises dans The Maximus Poems 10), Malick n'intègre que sur le plan de la thématique cet ancrage de la pensée dans les objets. En effet, c'est à travers une forme quelque peu naïve que le film retranscrit l'énergie qui porte les récits fondateurs des Etats-Unis $\mathbf{1 1}$.

Le dernier film de Malick illustre certains aspects de la pensée transcendantaliste en multipliant les allusions et les références à la culture des pionniers et leur relation à la nature. Notre analyse se limite à un pointage intertextuel qui ne prend pas en compte le hiatus entre la dimension "opératique» du film $\mathbf{1 2}$ d'une part, l'énonciation subjective de Thoreau, les rythmes variables des longs poèmes de Whitman et le scepticisme des essais d'Emerson d'autre part. John Smith et la princesse Pocahontas font figure de nouveaux Adam et Eve13 : celle-ci lui offre sa plume, sur la plage; tous deux communiquent tactilement, John Smith éprouvant la peau de la jeune fille, avant de lui passer la main sur le visage. Hymne de joie et ode à la nature, The New World traduit visuellement les chants de Whitman:

"Oh! faire un chant qui ne soit que de joies!

Plein de musique - plein de masculinité, de féminité, tout plein de l'enfance!

Plein de communes occupations - plein de grains et d'arbres.

Oh y mettre les voix des animaux - oh y mettre la vitesse et balance des poissons!

Oh dans un chant la tombée des gouttes de pluie!

Oh la lumière du soleil et le mouvement des vagues dans un chant! Oh la joie de mon esprit - il est hors de cage - il part comme l'éclair! »14

Sans craindre la démesure ni le kitsch, Malick s'attache à illustrer ces instants d'épiphanie, alternant entre couchers de soleil et gouttes de rosée qui perlent, élan de L'Or du Rhin et bruissement de la nature; le
9 William Carlos Williams, Paterson, New Directions, New York, 1963, p. 6 et p. 9 [première édition du "Book 1": 1946]. Voir aussi In the American Grain (New Directions, New York, 1956) sur le récit de la fondation du Nouveau Monde.

10 Charles Olson, The Maximus Poems, University of California Press, Berkeley/Los Angeles/ Londres, 1983 [1960-1975], pp. 53-55, pp. 7374, pp. $125-131$ et p. 554 .

11 Comme le note Jean Roy, The New World est "l'histoire d'une genèse", un "récit des origines", qui porte sur la fondation des EtatsUnis (L'Humanité, 15 février 2006).

12 The New World, tourné en $65 \mathrm{~mm}$ avec un budget de 30 millions de dollars, alterne paradoxalement une volonté de précision dans le rendu historique avec un lyrisme poétique, souligné à renfort de citations de L'Or du Rhin de Wagner sur la bande son (sur ce point, voir l'article de Laurent Guido dans le présent dossier). Le film, tourné en lumières naturelles, se déroule en Virginie, lieu effectif de l'accostage de John Smith en avril 1607. Le fort bâti par l'expédition est reconstruit avec un souci de véracité, tandis que les bateaux qui accostent sont d'authentiques pièces de musée. Si Malick mobilise comme référent des sources historiques, il n'en sacrifie pas moins à un mode de représentation irréalisant qui se manifeste symptomatiquement par l'assimilation du personnage féminin à une égérie de Hollywood.

13 Tout monde préservé de l'intervention humaine apparaît comme un paradis, pour qui sait goûter les miracles de la nature: "Le vent du matin souffle à jamais, le poème de la création est ininterrompu; mais rares sont les oreilles qui l'entendent. L'Olympe n'est que le dehors de la terre, partout." (Henry David Thoreau, Walden ou la vie dans les bois, L'Age d'Homme, Lausanne, 1985, p. 74 [traduit par Jeanne Chantal et Thierry Fournier]).

14 Walt Whitman, "Un chant de joies ", Poèmes. Feuilles d'herbe [1855], Gallimard, Paris, 1992, p. 99 [traduit par Louis Fabulet]. 
The New World, 2005

15 Une vie en communion avec le cycle des saisons restitue sa beauté originelle à l'homme, selon Whitman: "A présent si mille hommes parfaits devaient se présenter cela ne me surprendrait point, / A présent si mille beaux types de femmes se présentaient cela ne m'étonnerait point. / A présent je comprends le secret de fabrication des êtres supérieurs, / C'est de pousser en plein air et de manger et dormir avec la terre." (Walt Whitman, "Chant de la grand'route", Poèmes. Feuilles d'herbe, op. cit., p. 67).

16 Ralph Waldo Emerson, "La nature" [1836], La Confiance en soi et autres essais, Editions Payot/Rivages, Paris, 2000, pp. 21-23 [traduit par Monique Bégot].

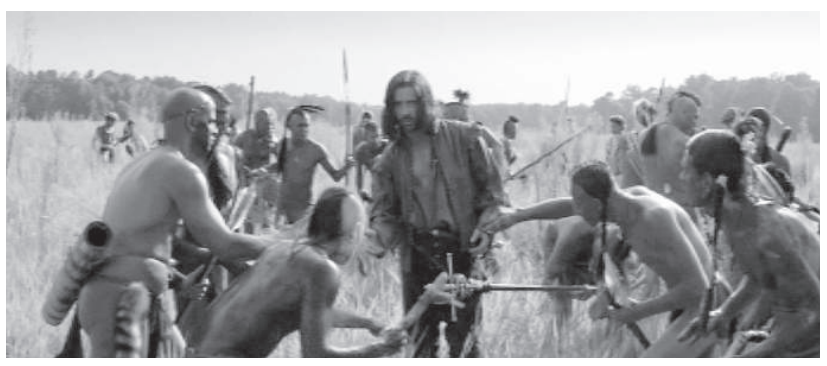

montage, relativement court et dispersif, et le cadrage, qui contribue à instaurer une focalisation multiple tout en multipliant les mouvements de caméra, soulignent le mouvement extatique du chant. En opposition au monde civilisé de l'ancienne Europe, The New World prône la vie au grand air comme un fortifiant et une sagesse philosophique, toujours en écho à Whitman ${ }^{15}$. Le corps et la nature sont explorés à travers la même dynamique désirante, en affirmant une «relation occulte entre l'homme et le règne végétal", conformément au panthéisme d'un Emerson. Rappelons en effet que cette confusion entre règnes fait système dans son célèbre essai sur la nature:

«A dire vrai, peu d'adultes savent voir la nature. La plupart des gens ne voient pas le soleil. Du moins en ont-ils une vue très superficielle. [...] En présence de la nature, l'homme est parcouru d'un sauvage frisson de délice, en dépit de la réalité de ses peines. [...] Debout sur le sol dénudé, la tête baignée par l'air vif, transporté par l'espace infini, tout égoïsme mesquin disparait. Je deviens un globe oculaire transparent; je ne suis rien; je vois tout; les courants de l'Etre universel circulent à travers moi; je fais partie intégrante de Dieu. [...] Le plus fort sentiment de délice que les champs et les bois procurent est de suggérer une relation occulte entre l'homme et le règne végétal.»16

John Smith fait l'expérience de cette communion avec les éléments naturels (le soleil, l'air, la terre), tandis que la variation des points de vue dans le film s'attache à restituer la vision omnipotente invoquée par Emerson, oscillant entre le regard de Dieu et un œil désincarné.

\section{Les idéogrammes du Nouveau Monde}

Lavenir et la nouveauté sont présentés dans The New World comme un retour au primitivisme, Malick investissant le cinéma comme un langage universel. En mettant en scène une langue archaïque, corporelle et visuelle, il réactive le mythe d'une pensée par l'image et d'une écriture idéogrammatique. Les Indiens s'expriment à travers une langue imagi- 
naire, chorégraphiée notamment à partir des pictogrammes des Algonquins. Le langage des gestes est valorisé par rapport à celui des mots - et le langage du film par rapport aux gravures et autres reconstitutions du Nouveau Monde, citées dans le générique. Lorsque la princesse indienne mime corporellement les éléments naturels (le ciel, le soleil, l'eau et le vent) avant de désigner et renommer les organes du visage aimé (les yeux, les lèvres et les oreilles), il est à vrai dire difficile de déterminer si c'est elle qui apprend l'anglais ou au contraire le capitaine qui est initié à un langage gestuel. Il est par contre indéniable que le film met en scène une langue d'avant le péché originel et la chute dans la rationalité, comme l'atteste la réflexion over de John Smith au sujet des indigènes:

"Ils sont doux. Affectueux. Fidèles. Exempts de toute fourberie. Les mots signifiant mensonge, tromperie, cupidité, envie, calomnie et pardon leur sont inconnus. Ils ne sont pas jaloux. Aucun sens de la possession."

L'intertexte emersonien est évident, celui-ci soutenant notamment dans La Nature que la parole vive et poétique porte trace des symboles primitifs :

"A cause de cette correspondance radicale entre les choses visibles et les pensées humaines, les êtres primitifs, qui ne possèdent que le nécessaire, conversent en symboles. [...] Cette dépendance immédiate du langage, par rapport à la nature, cette conversion d'un phénomène extérieur en un type qui trouve sa correspondance dans la vie de l'homme, ne perd jamais le pouvoir qu'elle a de nous affecter. C'est cela qui donne un tel piquant - que tout un chacun apprécie à la conversation d'un fermier au naturel vigoureux ou à celle d'un homme qui vit dans les bois. [...] La corruption de l'individu est suivie par la corruption du langage.»17

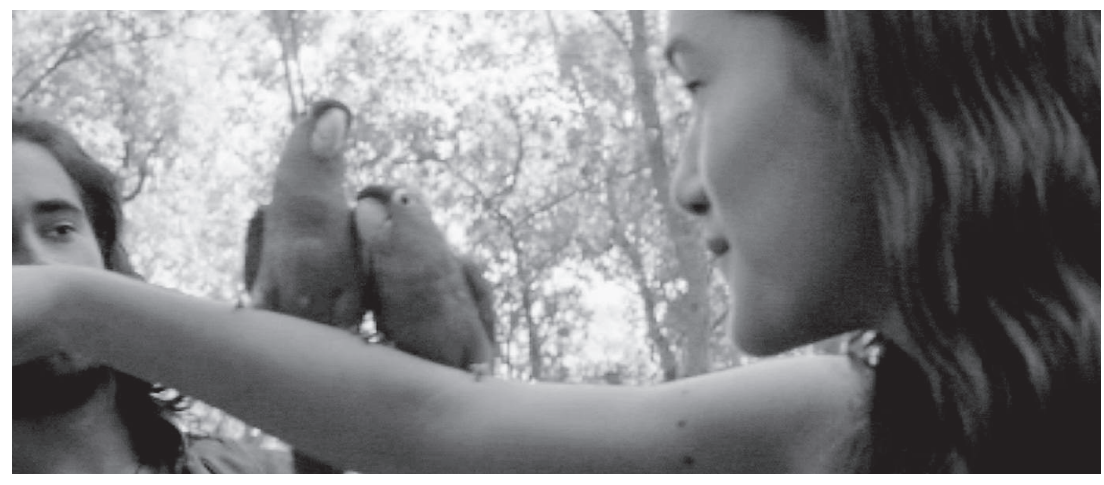

17 Ralph Waldo Emerson, "La nature", op. cit., pp. 39-40.

The New World, 2005 
18 A nouveau, le film évoque l'insouciance primitive promulguée par Thoreau: "Nous serions bien heureux si nous vivions toujours dans le présent et profitions de chaque incident qui nous arrive, comme l'herbe admet l'influence de la rosée la plus légère qui s'y dépose - et si nous ne passions pas notre temps à expier notre négligence lors des occasions passées, ce que nous appelons faire notre devoir." (Henry David Thoreau, Walden ou la vie dans les bois, op. cit., p. 271).
Et de fait, la jeune princesse Pocahontas est bannie par sa tribu, expulsée hors du paradis naturel, après s'être intégrée à la civilisation occidentale (notamment par le biais de l'apprentissage de l'anglais) - et avoir livré, tel Prométhée, des graines au camp retranché des Blancs.

\section{Au fil de l'eau}

L'eau qui s'écoule, le fleuve primitif sur lequel dériver valent comme la métonymie d'un élan vital. Mais le plan d'eau peut encore agir comme un miroir dans lequel se mirer, une représentation dans laquelle s'abîmer, en intervertissant les ordres du réel et de l'imaginaire. Le prologue de The New World met en jeu cette indistinction, jetant le trouble dans la représentation: l'eau reflète des arbres et des nuages, troublés par des gouttes de pluie; la princesse Pocahontas lève les bras au ciel, dans un geste d'incantation (voir l'analyse qu'Alain Boillat propose de ces deux plans inauguraux dans le présent dossier). Le motif de la surface d'eau apparaît comme un leitmotiv dans le film, en lien avec les aventures de John Smith et de la princesse Pocahontas dans la forêt. A la suite du générique, le film présente un couple d'indigènes nageant sous l'eau, comme en écho au début de The Thin Red Line; la caméra suit le couple, en les cadrant à travers différentes échelles de plan; à la faveur d'un fondu enchaîné, la surface d'eau est ressaisie depuis l'extérieur, trois navires sur le point d'accoster apparaissant lorsque la caméra s'élève. Ce motif est discrètement réinscrit lorsque John Smith s'intègre à la communauté indienne: l'eau claire d'une rivière s'écoule, marquant la renaissance du capitaine qui s'éveille à une vie primitive, résurrection soulignée par un gros plan sur un épi de blé et les réverbérations du soleil. Quelques minutes plus tard, le même motif réapparaît: la caméra fixe une surface d'eau avant d'amorcer un mouvement ascendant, découvrant des arbres qui se reflètent; dans le plan suivant, Smith, tenant un perroquet à son bras, fait face à la princesse en amorce, immédiatement intégrée au sein du cadre. Ces éléments pastoraux et idylliques culminent dans une séquence qui relie la thématique de l'amour à la nature nourricière et à l'abandon de soi $\mathbf{1 8}$. Le couple se tient au bord de la plage, Pocahontas rafraîchissant le visage de Smith à l'aide d'un seau d'eau. Sans transition, intervient une scène de danse rituelle, de nuit (la princesse Pocahontas invoque à nouveau l'économie du don: "Tout doit t'être donné»). Le motif de la surface d'eau, où se reflètent des arbres, apparaît à nouveau. Un plan d'ensemble présente la mer et la côte, ainsi qu'une nuée d'oiseaux qui traversent le ciel. En plan rapproché, la princesse est cadrée au sein d'une nature luxuriante, zébrée d'éclairs de chaleur. Confirmant ce mouvement de superposi- 
tion entre paysage et corps, les plans qui suivent cadrent un champ de blé, la princesse de dos, les cheveux au vent, et enfin le vol d'un oiseau. A travers de multiples cadrages, le couple est filmé enlacé. La séquence se clôt sur une surface d'eau; mais cette fois, les arbres, cadrés en contre-plongée, font l'objet d'un plan à part. Les principes masculin et féminin, les arbres et l'eau, tendent à se confondre, à se superposer.

L'eau, enfin, comme le montre bien la construction circulaire du film, fait le lien entre l'Ancien et le Nouveau Monde, la mer pouvant être franchie dans les deux sens. Aussi le premier mouvement du film, centré sur les aventures de John Smith et de la princesse Pocahontas, reproduit-il la structure de la quête et les aventures des pionniers. A cet égard, il est significatif que le capitaine Smith reparte seul pour de nouvelles conquêtes et colonisations, laissant la princesse indienne éplorée, en deuil. Le deuxième mouvement du film instaure un mode de vie sédentarisée, la princesse s'établissant avec John Rolfe. La nature, tout comme les impulsions de Pocahontas, sont dès lors dévitalisées: les jardins artificiels regardés avec incrédulité par un Indien qui a suivi la princesse en Angleterre, tout comme le labyrinthe que cette dernière parcourt en jouant avec son enfant, constituent le contre-champ de la vie dans les bois $\mathbf{1 9}$. Cette opposition entre deux rapports à la terre, redoublée par la dichotomie entre l'amour fou et les joies du mariage, se scelle par la maladie mortelle de la princesse contractée en Angleterre: pour la civilisation indienne, le voyage est sans retour.
19 Lors de l'ultime rencontre entre John Smith et Pocahontas, celui-ci affirme le rêve comme seule réalité, adhérant à la vie dans l'instant présent tant vantée par Whitman: "J'ai cru à un rêve ce que l'on a vécu dans la forêt. C'est la seule vérité. C'est comme si je te découvrais.". 